\title{
BOUNDARY OPERATORS IN QUANTUM FIELD THEORY
}

\author{
Giampiero Esposito \\ Istituto Nazionale di Fisica Nucleare, Sezione di Napoli, Complesso Universitario di Monte \\ S. Angelo, Via Cintia, Edificio N', 80126 Napoli, Italy \\ Università di Napoli Federico II, Dipartimento di Scienze Fisiche, Complesso Universitario \\ di Monte S. Angelo, Via Cintia, Edificio N', 80126 Napoli, Italy
}

The fundamental laws of physics can be derived from the requirement of invariance under suitable classes of transformations on the one hand, and from the need for a well-posed mathematical theory on the other hand. As a part of this programme, the present paper shows under which conditions the introduction of pseudo-differential boundary operators in one-loop Euclidean quantum gravity is compatible both with their invariance under infinitesimal diffeomorphisms and with the requirement of a strongly elliptic theory. Suitable assumptions on the kernel of the boundary operator make it therefore possible to overcome problems resulting from the choice of purely local boundary conditions.

PACS numbers: 03.70.+k, 04.70.Gw 


\section{Introduction}

The aim of theoretical physics is to provide a clear conceptual framework for the wide variety of natural phenomena, so that not only are we able to make accurate predictions to be checked against observations, but the underlying mathematical structures of the world we live in can also become sufficiently well understood by the scientific community. What are therefore the key elements of a mathematical description of the physical world? Can we derive all basic equations of theoretical physics from a few symmetry principles? What do they tell us about the origin and evolution of the universe? Why is gravitation so peculiar with respect to all other fundamental interactions?

The above questions have received careful consideration over the last decades, and have led, in particular, to several approaches to a theory aiming at achieving a synthesis of quantum physics on the one hand, and general relativity on the other hand. This remains, possibly, the most important task of theoretical physics. The need for a quantum theory of gravity is already clear from singularity theorems in classical cosmology. Such theorems [1] prove that the Einstein theory of general relativity leads to the occurrence of space-time singularities in a generic way. At first sight one might be tempted to conclude that a breakdown of all physical laws occurred in the past, or that general relativity is severely incomplete, being unable to predict what came out of a singularity. It has been therefore suggested that all these pathological features result from the attempt of using the Einstein theory well beyond its limit of validity, i.e. at energy scales where the fundamental theory is definitely more involved. General relativity might be therefore viewed as a low-energy limit of a richer theory, which achieves the synthesis of both the basic principles of modern physics and the fundamental interactions in the form presently known [2].

Within the framework just outlined it remains however true that the various approaches to quantum gravity developed so far suffer from mathematical inconsistencies, or incompleteness in their ability of accounting for some basic features of the laws of nature. From the point of view of general principles, the space-time approach to quantum mechanics and quantum field theory [3-5], and its application to the quantization of gravitational interactions, remains indeed of fundamental importance [6,7]. When one tries 
to implement the Feynman "sum over histories" one discovers that, already at the level of non-relativistic quantum mechanics, a well defined mathematical formulation is only obtained upon considering a heat-equation problem. The measure occurring in the Feynman representation of the Green kernel is then meaningful, and the propagation amplitude of quantum mechanics in flat Minkowski space-time is obtained by analytic continuation. This is a clear indication that quantum-mechanical problems via path integrals are well understood only if the heat-equation counterpart is mathematically well posed. In quantum field theory one then deals with the Euclidean approach, and its application to quantum gravity relies heavily on the theory of elliptic operators on Riemannian manifolds [8]. To obtain a complete picture one has then to specify the boundary conditions of the theory, i.e. the class of Riemannian geometries with their topologies involved in the sum, and the form of boundary data assigned on the bounding surfaces.

In particular, recent work [9] has shown that the only set of local boundary conditions on metric perturbations which are completely invariant under infinitesimal diffeomorphisms is incompatible with the request of a good elliptic theory. More precisely, while the resulting operator on metric perturbations can be made of Laplace type and elliptic in the interior of the Riemannian manifold under consideration, the property of strong ellipticity is violated. This is a precise mathematical expression of the request that a unique smooth solution of the boundary-value problem should exist which vanishes at infinite geodesic distance from the boundary. This opens deep interpretive issues, since only for gravity does the request of complete gauge invariance of the boundary conditions turn out to be incompatible with a good elliptic theory [9]. It is then impossible to make sense even just of the one-loop semiclassical approximation, because the functional trace of the heat operator is found to diverge [9].

We have been therefore led to consider non-local boundary conditions for the quantized gravitational field at one-loop level $[10,11]$. On the one hand, such a scheme already arises in simpler problems, i.e. the quantum theory of a free particle subject to non-local boundary data on a circle [12]. One then finds two families of eigenfunctions of the Hamiltonian: surface states which decrease exponentially as one moves away from the boundary, and bulk states which remain instead smooth and non-vanishing. The generalization to an Abelian 
gauge theory such as Maxwell theory can fulfill non-locality, ellipticity and complete gauge invariance of boundary conditions providing one learns to work with pseudo-differential operators in one-loop quantum theory [13]. On the other hand, in the application to quantum gravity, since the boundary operator acquires new kernels responsible for the pseudo-differential nature of the boundary-value problem, one might hope to be able to recover a good elliptic theory under a wider variety of conditions.

This is precisely the aim of the present paper. After a survey of operators of Laplace type and of the associated boundary operators in section 2, section 3 introduces integrodifferential boundary operators in Euclidean quantum gravity. Strong ellipticity of differential and pseudo-differential boundary-value problems is then defined in detail in section 4, and the application to Euclidean quantum gravity is studied in section 5. Further examples, of simpler nature, are given in section 6 , and concluding remarks are presented in section 7 .

\section{Operators of Laplace type and their boundary operators}

In the Euclidean approach to quantum field theory and quantum gravity one studies differentiable manifolds endowed with positive-definite metrics $g$, so that space-time is actually replaced by an $m$-dimensional Riemannian space $(M, g)$. An operator $\mathcal{P}$ of Laplace type, which acts on gauge fields, maps smooth sections of a vector bundle $V$ over $M$ into smooth sections of the same bundle, i.e.

$$
\mathcal{P}: C^{\infty}(V, M) \rightarrow C^{\infty}(V, M),
$$

and reads

$$
\mathcal{P}=-g^{a b} \nabla_{a} \nabla_{b}-E,
$$

where $g^{a b}$ is the contravariant form of the Riemannian metric for $M, \nabla$ is the connection on $V$, and $E$ is an endomorphism. In Ref. [9] a thorough investigation of boundary operators for elliptic operators of the form (2.1) has been performed. The key elements we need to recall are as follows. 
If the manifold $M$ has a smooth non-empty boundary $\partial M$, two vector bundles over $\partial M$, hereafter denoted by $W$ and $W^{\prime}$, yield a complete description of the problem. The boundary operator $B$ maps smooth sections of $W$ into smooth sections of $W^{\prime}$ :

$$
B: C^{\infty}(W, \partial M) \rightarrow C^{\infty}\left(W^{\prime}, \partial M\right) .
$$

For mixed boundary conditions, the operator $B$ frequently reads $[9,14,15]$

$$
B \equiv\left(\begin{array}{cc}
\Pi & 0 \\
\Lambda & I-\Pi
\end{array}\right)
$$

where $\Pi$ and $I-\Pi$ are complementary projectors, and $\Lambda$ is a first-order tangential differential operator

$$
\Lambda \equiv(I-\Pi)\left[\frac{1}{2}\left(\Gamma^{i} \widehat{\nabla}_{i}+\widehat{\nabla}_{i} \Gamma^{i}\right)+S\right](I-\Pi) .
$$

With our notation, $\Gamma^{i}$ are endomorphism-valued vector fields on the boundary, $\widehat{\nabla}$ is the induced connection on $\partial M$, and $S$ is an endomorphism on $\partial M$. By virtue of (2.3) one has

$$
\Pi \Lambda=\Lambda \Pi=0,
$$

and hence $B$ is a projector, in that $B^{2}=B$. The boundary-value problem is meant to be the pair $(\mathcal{P}, B)$, where $\mathcal{P}$ is the operator (2.1) and $B$ is given in (2.2). The corresponding mixed boundary conditions read

$$
\left(\begin{array}{cc}
\Pi & 0 \\
\Lambda & I-\Pi
\end{array}\right)\left(\begin{array}{c}
{[\varphi]_{\partial M}} \\
{\left[\varphi_{; N}\right]_{\partial M}}
\end{array}\right)=0
$$

where ${ }_{; N}$ denotes covariant differentiation along the direction normal to the boundary, i.e. $N^{a} \nabla_{a}$. Moreover, the boundary operator (2.2) may be expressed in the form

$$
B=P L,
$$

where $P$ is the map

$$
P: C^{\infty}(W, \partial M) \rightarrow C^{\infty}\left(W^{\prime}, \partial M\right)
$$


given by

$$
P \equiv\left(\begin{array}{cc}
\Pi & 0 \\
0 & I-\Pi
\end{array}\right)
$$

and $L$ is a map

$$
L: C^{\infty}(W, \partial M) \rightarrow C^{\infty}(W, \partial M)
$$

expressed in matrix form as

$$
L \equiv\left(\begin{array}{cc}
I & 0 \\
\Lambda & I
\end{array}\right)
$$

Interestingly, the operator $P$ is itself a projector: $P^{2}=P$, whereas $L$ is non-singular, with inverse

$$
L^{-1}=\left(\begin{array}{cc}
I & 0 \\
-\Lambda^{-1} & I
\end{array}\right) .
$$

The "column vector" used in Eq. (2.5), i.e.

$$
\psi(\varphi) \equiv\left(\begin{array}{c}
{[\varphi]_{\partial M}} \\
{[\varphi ; N]_{\partial M}}
\end{array}\right)
$$

is a section of the bundle $W$ of boundary data, whereas the auxiliary vector bundle $W^{\prime}$ has sections given by (see the main diagonal of $B$ in $(2.2)$ )

$$
\psi^{\prime}(\varphi) \equiv\left(\begin{array}{c}
\Pi[\varphi]_{\partial M} \\
(I-\Pi)\left[\varphi_{; N}\right]_{\partial M}
\end{array}\right)
$$

At this stage, a naturally occurring question is under which conditions a projector $P$ gives rise to a projector $B$ such that $B=P L$ as in Eq. (2.6). To obtain equations in a form as general as possible we replace $P$ defined in $(2.7)$ by the $2 \times 2$ matrix

$$
P \equiv\left(\begin{array}{ll}
\alpha & \beta \\
\gamma & \delta
\end{array}\right)
$$

where $\alpha, \beta, \gamma, \delta$ are, for the time being, some unknown operators to be determined by imposing suitable restrictions (see below). The projector condition $P^{2}=P$ yields therefore four operator equations, i.e.

$$
\alpha^{2}+\beta \gamma=\alpha
$$




$$
\begin{aligned}
& \alpha \beta+\beta \delta=\beta \\
& \gamma \alpha+\delta \gamma=\gamma \\
& \gamma \beta+\delta^{2}=\delta
\end{aligned}
$$

A particular solution of Eqs. (2.13)-(2.16) is given by the case in which

$$
\begin{gathered}
\beta=\gamma=0, \\
\alpha^{2}=\alpha, \\
\delta^{2}=\delta, \\
\alpha+\delta=I .
\end{gathered}
$$

This yields the operator $P$ in the form (2.7) appropriate for the Grubb-Gilkey-Smith boundary-value problem $[14,15]$. If the conditions $(2.17)-(2.20)$ are not fulfilled, one gets instead from Eqs. (2.13)-(2.16) the equations

$$
\begin{gathered}
\alpha(\alpha-I)=-\beta \gamma \\
\alpha=I-\beta \delta \beta^{-1}, \\
\alpha=I-\gamma^{-1} \delta \gamma \\
\delta(\delta-I)=-\gamma \beta
\end{gathered}
$$

provided that $\beta$ and $\gamma$ can be inverted.

\section{Euclidean quantum gravity}

In Euclidean quantum gravity, mixed boundary conditions on metric perturbations $h_{c d}$ occur naturally if one requires their complete invariance under infinitesimal diffeomorphisms, as is proved in detail in Ref. [9]. On denoting by $N^{a}$ the inward-pointing unit normal to the boundary, by

$$
q_{b}^{a} \equiv \delta_{b}^{a}-N^{a} N_{b}
$$


the projector of tensor fields onto $\partial M$, with associated projection operator

$$
\Pi_{a b}^{c d} \equiv q_{(a}^{c} q_{b)}^{d}
$$

the gauge-invariant boundary conditions for one-loop quantum gravity read [9]

$$
\begin{gathered}
{\left[\Pi_{a b}^{c d} h_{c d}\right]_{\partial M}=0} \\
{\left[\Phi_{a}(h)\right]_{\partial M}=0}
\end{gathered}
$$

where $\Phi_{a}$ is the gauge-averaging functional necessary to obtain an invertible operator $P_{a b}{ }^{c d}$ on metric perturbations. When $P_{a b}{ }^{c d}$ is chosen to be of Laplace type, $\Phi_{a}$ reduces to the familiar de Donder term

$$
\Phi_{a}(h)=\nabla^{b}\left(h_{a b}-\frac{1}{2} g_{a b} g^{c d} h_{c d}\right)=E_{a}^{b c d} \nabla_{b} h_{c d}
$$

where $E^{a b c d}$ is the DeWitt supermetric on the vector bundle of symmetric rank-two tensor fields over $M(g$ being the metric on $M)$ :

$$
E^{a b c d} \equiv \frac{1}{2}\left(g^{a c} g^{b d}+g^{a d} g^{b c}-g^{a b} g^{c d}\right)
$$

The boundary conditions (3.3) and (3.4) can then be cast in the Grubb-Gilkey-Smith form (2.5), where $\Lambda$ is the first-order operator on the boundary defined in Eq. (2.3). However, the work in Ref. [9] has shown that an operator of Laplace type on metric perturbations is then incompatible with the requirement of strong ellipticity of the boundary-value problem (see section 4), because the operator $\Lambda$ contains tangential derivatives of metric perturbations.

To take care of this serious drawback, the work in Refs. [10,11] has proposed to consider in the boundary condition (3.4) a gauge-averaging functional given by the de Donder term (3.5) plus an integro-differential operator on metric perturbations, i.e.

$$
\Phi_{a}(h) \equiv E_{a}^{b c d} \nabla_{b} h_{c d}+\int_{M} \zeta_{a}^{c d}\left(x, x^{\prime}\right) h_{c d}\left(x^{\prime}\right) d V^{\prime}
$$


We now begin by remarking that the resulting boundary conditions can be cast in the form

$$
\left(\begin{array}{cc}
\Pi & 0 \\
\Lambda+\widetilde{\Lambda} & I-\Pi
\end{array}\right)\left(\begin{array}{c}
{[\varphi]_{\partial M}} \\
{[\varphi ; N]_{\partial M}}
\end{array}\right)=0
$$

where $\widetilde{\Lambda}$ reflects the occurrence of the integral over $M$ in Eq. (3.7). It is convenient to work first in a general way and then consider the form taken by these operators in the gravitational case. On requiring that the resulting boundary operator

$$
\mathcal{B}=\left(\begin{array}{ll}
\mathcal{B}_{11} & \mathcal{B}_{12} \\
\mathcal{B}_{21} & \mathcal{B}_{22}
\end{array}\right) \equiv\left(\begin{array}{cc}
\Pi & 0 \\
\Lambda+\widetilde{\Lambda} & I-\Pi
\end{array}\right)
$$

should remain a projector: $\mathcal{B}^{2}=\mathcal{B}$, we find the condition

$$
(\Lambda+\widetilde{\Lambda}) \Pi-\Pi(\Lambda+\widetilde{\Lambda})=0
$$

which reduces to

$$
\Pi \widetilde{\Lambda}=\widetilde{\Lambda} \Pi
$$

by virtue of (2.4).

In Euclidean quantum gravity at one-loop level, Eq. (3.11) leads to

$$
\Pi_{a}^{b} \underset{c}{r}(x) \int_{M} \zeta_{b}^{c q}\left(x, x^{\prime}\right) h_{q r}\left(x^{\prime}\right) d V^{\prime}=\int_{M} \zeta_{a}^{c d}\left(x, x^{\prime}\right) \Pi_{c d}^{q r}\left(x^{\prime}\right) h_{q r}\left(x^{\prime}\right) d V^{\prime}
$$

which can be re-expressed in the form

$$
\int_{M}\left[\Pi_{a}^{b}{ }_{c}^{r}(x) \zeta_{b}^{c q}\left(x, x^{\prime}\right)-\zeta_{a}^{c d}\left(x, x^{\prime}\right) \Pi_{c d}^{q r}\left(x^{\prime}\right)\right] h_{q r}\left(x^{\prime}\right) d V^{\prime}=0
$$

Since this should hold for all $h_{q r}\left(x^{\prime}\right)$, it eventually leads to the vanishing of the term in square brackets in the integrand. The notation $\zeta_{b}^{c q}\left(x, x^{\prime}\right)$ is indeed rather awkward, because there is an even number of arguments, i.e. $x$ and $x^{\prime}$, with an odd number of indices. Hereafter, we therefore assume that a vector field $T$ and kernel $\widetilde{\zeta}$ exist such that

$$
\zeta_{b}^{c q}\left(x, x^{\prime}\right) \equiv T^{p}(x) \widetilde{\zeta}_{b p}^{c q}\left(x, x^{\prime}\right) \equiv T^{p} \widetilde{\zeta}_{b p}{ }^{c} q^{\prime}
$$


The projector condition (3.11) is therefore satisfied if and only if

$$
T^{p}(x)\left[\Pi_{a}^{b} r_{c}^{r}(x) \widetilde{\zeta}_{b p}^{c q}\left(x, x^{\prime}\right)-\widetilde{\zeta}_{a p}^{c d}\left(x, x^{\prime}\right) \Pi_{c d}^{q r}\left(x^{\prime}\right)\right]=0
$$

\section{Strong ellipticity}

We are here concerned with the issue of ellipticity of the boundary-value problem of

section 3. For this purpose, we begin by recalling what is known about ellipticity of the Laplacian (hereafter $P$ ) on a Riemannian manifold with smooth boundary. This concept is studied in terms of the leading symbol of $P$. It is indeed well known that the Fourier transform makes it possible to associate to a differential operator of order $k$ a polynomial of degree $k$, called the characteristic polynomial or symbol. The leading symbol, $\sigma_{L}$, picks out the highest order part of this polynomial. For the Laplacian, it reads

$$
\sigma_{L}(P ; x, \xi)=|\xi|^{2} I=g^{\mu \nu} \xi_{\mu} \xi_{\nu} I
$$

With a standard notation, $(x, \xi)$ are local coordinates for $T^{*}(M)$, the cotangent bundle of $M$. The leading symbol of $P$ is trivially elliptic in the interior of $M$, since the right-hand side of (4.1) is positive-definite, and one has

$$
\operatorname{det}\left[\sigma_{L}(P ; x, \xi)-\lambda\right]=\left(|\xi|^{2}-\lambda\right)^{\operatorname{dim} V} \neq 0
$$

for all $\lambda \in \mathcal{C}-\mathbf{R}_{+}$. In the presence of a boundary, however, one needs a more careful definition of ellipticity. First, for a manifold $M$ of dimension $m$, the $m$ coordinates $x$ are split into $m-1$ local coordinates on $\partial M$, hereafter denoted by $\left\{\hat{x}^{k}\right\}$, and $r$, the geodesic distance to the boundary. Moreover, the $m$ coordinates $\xi_{\mu}$ are split into $m-1$ coordinates $\left\{\zeta_{j}\right\}$ (with $\zeta$ being a cotangent vector on the boundary), jointly with a real parameter $\omega \in T^{*}(\mathbf{R})$. At a deeper level, all this reflects the split

$$
T^{*}(M)=T^{*}(\partial M) \oplus T^{*}(\mathbf{R})
$$

in a neighbourhood of the boundary $[8,9]$. 
The ellipticity we are interested in requires now that $\sigma_{L}$ should be elliptic in the interior of $M$, as specified before, and that strong ellipticity should hold. This means that a unique solution exists of the differential equation obtained from the leading symbol:

$$
\left[\sigma_{L}\left(P ;\left\{\hat{x}^{k}\right\}, r=0,\left\{\zeta_{j}\right\}, \omega \rightarrow-i \frac{\partial}{\partial r}\right)-\lambda\right] \varphi(r, \hat{x}, \zeta ; \lambda)=0
$$

subject to the boundary conditions

$$
\sigma_{g}(B)\left(\left\{\hat{x}^{k}\right\},\left\{\zeta_{j}\right\}\right) \psi(\varphi)=\psi^{\prime}(\varphi)
$$

and to the asymptotic condition

$$
\lim _{r \rightarrow \infty} \varphi(r, \hat{x}, \zeta ; \lambda)=0
$$

In Eq. (4.5), $\sigma_{g}$ is the graded leading symbol of the boundary operator of section 2 in the local coordinates $\left\{\hat{x}^{k}\right\},\left\{\zeta_{j}\right\}$, and is given by

$$
\sigma_{g}(B)=\left(\begin{array}{cc}
\Pi & 0 \\
i \Gamma^{j} \zeta_{j} & I-\Pi
\end{array}\right)
$$

Roughly speaking, the above construction uses Fourier transform and the inward geodesic flow to obtain the ordinary differential equation (4.4) from the Laplacian, with corresponding Fourier transform (4.5) of the original boundary conditions. The asymptotic condition (4.6) picks out the solutions of Eq. (4.4) which satisfy Eq. (4.5) with arbitrary boundary data $\psi^{\prime}(\varphi)$ (see (2.11)) and vanish at infinite geodesic distance to the boundary. When all the above conditions are satisfied $\forall \zeta \in T^{*}(\partial M), \forall \lambda \in \mathcal{C}-\mathbf{R}_{+}, \forall(\zeta, \lambda) \neq(0,0)$ and $\forall \psi^{\prime}(\varphi) \in C^{\infty}\left(W^{\prime}, \partial M\right)$, the boundary-value problem $(P, B)$ for the Laplacian is said to be strongly elliptic with respect to the cone $\mathcal{C}-\mathbf{R}_{+}$.

However, when the gauge-averaging functional (3.7) is used in the boundary condition (3.4), the work in Ref. [11] has proved that the operator on metric perturbations takes the form of an operator of Laplace type $P_{a b}{ }^{c d}$ plus an integral operator $G_{a b}{ }^{c d}$. Explicitly, one finds [11] (with $R_{b c d}^{a}$ being the Riemann curvature of the background geometry $(M, g)$ )

$$
P_{a b}{ }^{c d}=E_{a b}{ }^{c d}(-\square+R)-2 E_{a b}^{q f} R_{q p f}^{c} g^{d p}-E_{a b}{ }^{p d} R_{p}^{c}-E_{a b}{ }^{c p} R_{p}^{d},
$$




$$
G_{a b}^{c d}=U_{a b}^{c d}+V_{a b}^{c d}
$$

where

$$
\begin{gathered}
U_{a b}{ }^{c d} h_{c d}(x)=-2 E_{r s a b} \nabla^{r} \int_{M} T^{p}(x) \widetilde{\zeta}_{p}^{s}{ }^{c d}\left(x, x^{\prime}\right) h_{c d}\left(x^{\prime}\right) d V^{\prime}, \\
h^{a b} V_{a b}{ }^{c d} h_{c d}(x)=\int_{M^{2}} h^{a b}\left(x^{\prime}\right) T^{q}(x) \widetilde{\zeta}_{p q a b}\left(x, x^{\prime}\right) T^{r}(x) \widetilde{\zeta}_{r}^{p}{ }^{c d}\left(x, x^{\prime \prime}\right) h_{c d}\left(x^{\prime \prime}\right) d V^{\prime} d V^{\prime \prime} .
\end{gathered}
$$

We now assume that the operator on metric perturbations, which is so far an integrodifferential operator defined by a kernel, is also pseudo-differential. This means that it can be characterized by suitable regularity properties obeyed by the symbol. More precisely, let $S^{d}$ be the set of all symbols $p(x, \xi)$ such that [8]

(1) $p$ is $C^{\infty}$ in $(x, \xi)$, with compact $x$ support.

(2) For all $(\alpha, \beta)$, there exist constants $C_{\alpha, \beta}$ for which

$$
\begin{aligned}
& \left|(-i)^{\sum_{k=1}^{m}\left(\alpha_{k}+\beta_{k}\right)}\left(\frac{\partial}{\partial x_{1}}\right)^{\alpha_{1}} \ldots\left(\frac{\partial}{\partial x_{m}}\right)^{\alpha_{m}}\left(\frac{\partial}{\partial \xi_{1}}\right)^{\beta_{1}} \ldots\left(\frac{\partial}{\partial \xi_{m}}\right)^{\beta_{m}} p(x, \xi)\right| \\
& \leq C_{\alpha, \beta}\left(1+\sqrt{g^{a b}(x) \xi_{a} \xi_{b}}\right)^{d-\sum_{k=1}^{m} \beta_{k}},
\end{aligned}
$$

for some real (not necessarily positive) value of $d$. The associated pseudo-differential operator, defined on the Schwarz space and taking values in the set of smooth functions on $M$ with compact support:

$$
P: \mathcal{S} \rightarrow C_{c}^{\infty}(M)
$$

acts according to

$$
P f(x) \equiv \int e^{i(x-y) \cdot \xi} p(x, \xi) f(y) \mu(y, \xi)
$$

where $\mu(y, \xi)$ is here meant to be the invariant integration measure with respect to $y_{1}, \ldots, y_{m}$ and $\xi_{1}, \ldots, \xi_{m}$. Actually, one first gives the definition for pseudo-differential operators $P: \mathcal{S} \rightarrow C_{c}^{\infty}\left(\mathbf{R}^{m}\right)$, eventually proving that a coordinate-free definition can be given and extended to smooth Riemannian manifolds [8]. 
In the presence of pseudo-differential operators, both ellipticity in the interior of $M$ and strong ellipticity of the boundary-value problem need a more involved formulation. In our paper, inspired by the flat-space analysis in Ref. [16], we make the following requirements.

\subsection{Ellipticity in the interior}

Let $U$ be an open subset with compact closure in $M$, and consider an open subset $U_{1}$ whose closure $\bar{U}_{1}$ is properly included into $U: \bar{U}_{1} \subset U$. If $p$ is a symbol of order $d$ on $U$,

it is said to be elliptic on $U_{1}$ if there exists an open set $U_{2}$ which contains $\bar{U}_{1}$ and positive constants $C_{0}, C_{1}$ so that

$$
|p(x, \xi)|^{-1} \leq C_{1}(1+|\xi|)^{-d},
$$

for $|\xi| \geq C_{0}$ and $x \in U_{2}$, where $|\xi| \equiv \sqrt{g^{a b}(x) \xi_{a} \xi_{b}}$. The corresponding operator $P$ is then elliptic.

\subsection{Strong ellipticity in the absence of boundaries}

Let us assume that the symbol under consideration is polyhomogeneous, in that it admits an asymptotic expansion of the form

$$
p(x, \xi) \sim \sum_{l=0}^{\infty} p_{d-l}(x, \xi),
$$

where each term $p_{d-l}$ has the homogeneity property [16]

$$
p_{d-l}(x, t \xi)=t^{d-l} p_{d-l}(x, \xi) \text { if } t \geq 1 \text { and }|\xi| \geq 1
$$

The leading symbol is then, by definition,

$$
p^{0}(x, \xi) \equiv p_{d}(x, \xi) .
$$

Strong ellipticity in the absence of boundaries is formulated in terms of the leading symbol, and it requires that

$$
\operatorname{Re} p^{0}(x, \xi) \geq c(x)|\xi|^{d},
$$


where $x \in M$ and $|\xi| \geq 1, c$ being a positive function on $M$. It can then be proved that the Gärding inequality holds, according to which, for any $\varepsilon>0$,

$$
\operatorname{Re}(P u, u) \geq b\|u\|_{\frac{d}{2}}^{2}-b_{1}\|u\|_{\frac{d}{2}-\varepsilon}^{2} \text { for } u \in H^{\frac{d}{2}}(M)
$$

with $b>0$, where $H^{s}(M)$ is the standard notation for Sobolev spaces, for all $s[8,16]$.

\subsection{Strong ellipticity in the presence of boundaries}

The homogeneity property (4.16) only holds for $t \geq 1$ and $|\xi| \geq 1$. Consider now the case $l=0$, for which one obtains the leading symbol which plays the key role in the definition

of ellipticity. If $p^{0}(x, \xi) \equiv p_{d}(x, \xi) \equiv \sigma_{L}(P ; x, \xi)$ is not a polynomial (which corresponds to the genuinely pseudo-differential case) while being a homogeneous function of $\xi$, it is irregular at $\xi=0$. When $|\xi| \leq 1$, the only control over the leading symbol is provided by estimates of the form [16]

$$
\begin{aligned}
& \left|(-i)^{\sum_{k=1}^{m}\left(\alpha_{k}+\beta_{k}\right)}\left(\frac{\partial}{\partial x_{1}}\right)^{\alpha_{1}} \ldots\left(\frac{\partial}{\partial x_{m}}\right)^{\alpha_{m}}\left(\frac{\partial}{\partial \xi_{1}}\right)^{\beta_{1}} \ldots\left(\frac{\partial}{\partial \xi_{m}}\right)^{\beta_{m}} p^{0}(x, \xi)\right| \\
& \leq c(x)\langle\xi\rangle^{d-|\beta|} .
\end{aligned}
$$

We therefore come to appreciate the problematic aspect of symbols of pseudo-differential operators [16]. The singularity at $\xi=0$ can be dealt with either by modifying the leading symbol for small $\xi$ to be a $C^{\infty}$ function (at the price of loosing the homogeneity there), or by keeping the strict homogeneity and dealing with the singularity at $\xi=0$ [16].

On the other hand, we are interested in a definition of strong ellipticity of pseudodifferential boundary-value problems that reduces to Eqs. (4.4)-(4.6) when both $P$ and the boundary operator reduce to the form considered in section 2. For this purpose, and bearing in mind the occurrence of singularities in the leading symbols of $P$ and of the boundary operator, we make the following requirements.

Let $(P+G)$ be a pseudo-differential operator subject to boundary conditions described by the pseudo-differential boundary operator $\mathcal{B}$ (the consideration of $(P+G)$ rather than only $P$ is necessary to achieve self-adjointness, as is described in detail in Refs. [16] and 
[10]). The pseudo-differential boundary-value problem $((P+G), \mathcal{B})$ is strongly elliptic with respect to $\mathcal{C}-\mathbf{R}_{+}$if:

(I) The inequalities (4.14) and (4.18) hold;

(II) There exists a unique solution of the equation

$$
\left[\sigma_{L}\left((P+G) ;\left\{\hat{x}^{k}\right\}, r=0,\left\{\zeta_{j}\right\}, \omega \rightarrow-i \frac{\partial}{\partial r}\right)-\lambda\right] \varphi(r, \hat{x}, \zeta ; \lambda)=0
$$

subject to the boundary conditions

$$
\sigma_{L}(\mathcal{B})\left(\left\{\hat{x}^{k}\right\},\left\{\zeta_{j}\right\}\right) \psi(\varphi)=\psi^{\prime}(\varphi)
$$

and to the asymptotic condition (4.6). It should be stressed that, unlike the case of differential operators, Eq. (4.4') is not an ordinary differential equation in general, because $(P+G)$ is pseudo-differential.

(III) The strictly homogeneous symbols associated to $(P+G)$ and $\mathcal{B}$ have limits for $|\zeta| \rightarrow 0$ in the respective leading symbol norms, with the limiting symbol restricted to the boundary which avoids the values $\lambda \notin \mathcal{C}-\mathbf{R}_{+}$for all $\{\hat{x}\}$.

Condition (III) requires a last effort for a proper understanding. Given a pseudodifferential operator of order $d$ with leading symbol $p^{0}(x, \xi)$, the associated strictly homogeneous symbol is defined by [16]

$$
p^{h}(x, \xi) \equiv|\xi|^{d} p^{0}\left(x, \frac{\xi}{|\xi|}\right) \text { for } \xi \neq 0
$$

This extends to a continuous function vanishing at $\xi=0$ when $d>0$. In the presence of boundaries, the boundary-value problem $((P+G), \mathcal{B})$ has a strictly homogeneous symbol on the boundary equal to (some indices are omitted for simplicity)

$$
\left(\begin{array}{c}
p^{h}\left(\{\hat{x}\}, r=0,\{\zeta\},-i \frac{\partial}{\partial r}\right)+g^{h}\left(\{\hat{x}\},\{\zeta\},-i \frac{\partial}{\partial r}\right)-\lambda \\
b^{h}\left(\{\hat{x}\},\{\zeta\},-i \frac{\partial}{\partial r}\right)
\end{array}\right),
$$


where $p^{h}, g^{h}$ and $b^{h}$ are the strictly homogeneous symbols of $P, G$ and $\mathcal{B}$ respectively, obtained from the corresponding leading symbols $p^{0}, g^{0}$ and $b^{0}$ via equations analogous to (4.21), after taking into account the split (4.3), and upon replacing $\omega$ by $-i \frac{\partial}{\partial r}$. The limiting symbol restricted to the boundary (also called limiting $\lambda$-dependent boundary symbol operator) and mentioned in condition III reads therefore [16]

$$
\begin{aligned}
& a^{h}\left(\{\hat{x}\}, r=0, \zeta=0,-i \frac{\partial}{\partial r}\right) \\
& =\left(\begin{array}{c}
p^{h}\left(\{\hat{x}\}, r=0, \zeta=0,-i \frac{\partial}{\partial r}\right)+g^{h}\left(\{\hat{x}\}, \zeta=0,-i \frac{\partial}{\partial r}\right)-\lambda \\
b^{h}\left(\{\hat{x}\}, \zeta=0,-i \frac{\partial}{\partial r}\right)
\end{array}\right),
\end{aligned}
$$

where the singularity at $\xi=0$ of the leading symbol in absence of boundaries is replaced by the singularity at $\zeta=0$ of the leading symbols of $P, G$ and $\mathcal{B}$ when a boundary occurs.

\section{Application of the strong ellipticity criterion}

Let us now see how the previous conditions on the leading symbol of $(P+G)$ and on the graded leading symbol of the boundary operator can be used. The equation (4.4') is solved by a function $\varphi$ depending on $r,\left\{\hat{x}^{k}\right\},\left\{\zeta_{j}\right\}$ and, parametrically, on the eigenvalues $\lambda$. For simplicity, we write $\varphi=\varphi(r, \hat{x}, \zeta ; \lambda)$, omitting indices. Since the leading symbol is no longer a polynomial when $(P+G)$ is genuinely pseudo-differential, we cannot make any further specification on $\varphi$ at this stage, apart from requiring that it should reduce to (here $|\zeta|^{2} \equiv \zeta_{i} \zeta^{i}$ )

$$
\chi(\hat{x}, \zeta) e^{-r \sqrt{|\zeta|^{2}-\lambda}}
$$

when $(P+G)$ reduces to a Laplacian (and hence $\Lambda$ reduces to (2.3)).

The equation (4.5') involves the graded leading symbol of $\mathcal{B}$ and restrictions to the boundary of the field and its covariant derivative along the normal direction. Such a restriction is obtained by setting to zero the geodesic distance $r$, and hence we write, in general form (here we denote again by $\Lambda$ the full matrix element $\mathcal{B}_{21}$ in the boundary operator (3.9)),

$$
\left(\begin{array}{cc}
\Pi & 0 \\
\sigma_{L}(\Lambda) & I-\Pi
\end{array}\right)\left(\begin{array}{c}
\varphi(0, \hat{x}, \zeta ; \lambda) \\
\varphi^{\prime}(0, \hat{x}, \zeta ; \lambda)
\end{array}\right)=\left(\begin{array}{c}
\Pi \rho(0, \hat{x}, \zeta ; \lambda) \\
(I-\Pi) \rho^{\prime}(0, \hat{x}, \zeta ; \lambda)
\end{array}\right)
$$


where $\rho$ differs from $\varphi$, because Eq. (4.5') is written for $\psi(\varphi)$ and $\psi^{\prime}(\varphi) \neq \psi(\varphi)$. Now Eq. (5.1) leads to

$$
\begin{gathered}
\Pi \varphi(0, \hat{x}, \zeta ; \lambda)=\Pi \rho(0, \hat{x}, \zeta ; \lambda) \\
\sigma_{L}(\Lambda) \varphi(0, \hat{x}, \zeta ; \lambda)+(I-\Pi) \varphi^{\prime}(0, \hat{x}, \zeta ; \lambda)=(I-\Pi) \rho^{\prime}(0, \hat{x}, \zeta ; \lambda),
\end{gathered}
$$

and we require that, for $\varphi$ solving Eq. (4.4') and the asymptotic decay (4.6), with $\lambda \in$ $\mathcal{C}-\mathbf{R}_{+}$, Eqs. (5.2) and (5.3) can be always solved with given values of $\rho(0, \hat{x}, \zeta ; \lambda)$ and $\rho^{\prime}(0, \hat{x}, \zeta ; \lambda)$, whenever $(\zeta, \lambda) \neq(0,0)$. The idea is now to relate, if possible, $\varphi^{\prime}(0, \hat{x}, \zeta ; \lambda)$ to $\varphi(0, \hat{x}, \zeta ; \lambda)$ in such a way that Eq. (5.2) can be used to simplify Eq. (5.3). For this purpose, we consider the function $f$ such that

$$
\begin{gathered}
\frac{\varphi^{\prime}(0, \hat{x}, \zeta ; \lambda)}{\varphi(0, \hat{x}, \zeta ; \lambda)}=\frac{\rho^{\prime}(0, \hat{x}, \zeta ; \lambda)}{\rho(0, \hat{x}, \zeta ; \lambda)}=f(\hat{x}, \zeta ; \lambda), \\
\Pi(\hat{x}) f(\hat{x}, \zeta ; \lambda)=f(\hat{x}, \zeta ; \lambda) \Pi(\hat{x}) .
\end{gathered}
$$

If both (5.4) and (5.5) hold, Eq. (5.3) reduces indeed to

$$
\begin{aligned}
& \sigma_{L}(\Lambda) \varphi(0, \hat{x}, \zeta ; \lambda)+f(\hat{x}, \zeta ; \lambda)(\varphi(0, \hat{x}, \zeta ; \lambda)-\rho(0, \hat{x}, \zeta ; \lambda)) \\
& =f(\hat{x}, \zeta ; \lambda) \Pi(\varphi(0, \hat{x}, \zeta ; \lambda)-\rho(0, \hat{x}, \zeta ; \lambda))
\end{aligned}
$$

and hence, by virtue of (5.2),

$$
\left[\sigma_{L}(\Lambda)+f(\hat{x}, \zeta ; \lambda)\right] \varphi(0, \hat{x}, \zeta ; \lambda)=\rho^{\prime}(0, \hat{x}, \zeta ; \lambda)
$$

Thus, the strong ellipticity condition with respect to $\mathcal{C}-\mathbf{R}_{+}$implies in this case the invertibility of $\left[\sigma_{L}(\Lambda)+f(\hat{x}, \zeta ; \lambda)\right]$, i.e.

$$
\operatorname{det}\left[\sigma_{L}(\Lambda)+f(\hat{x}, \zeta ; \lambda)\right] \neq 0 \quad \forall \lambda \in \mathcal{C}-\mathbf{R}_{+}
$$

Moreover, by virtue of the identity

$$
\left[f(\hat{x}, \zeta ; \lambda)+\sigma_{L}(\Lambda)\right]\left[f(\hat{x}, \zeta ; \lambda)-\sigma_{L}(\Lambda)\right]=\left[f^{2}(\hat{x}, \zeta ; \lambda)-\sigma_{L}^{2}(\Lambda)\right]
$$


the condition (5.7) is equivalent to

$$
\operatorname{det}\left[f^{2}(\hat{x}, \zeta ; \lambda)-\sigma_{L}^{2}(\Lambda)\right] \neq 0 \quad \forall \lambda \in \mathcal{C}-\mathbf{R}_{+}
$$

Since $f(\hat{x}, \zeta ; \lambda)$ is, in general, complex-valued, one can always express it in the form

$$
f(\hat{x}, \zeta ; \lambda)=\operatorname{Re} f(\hat{x}, \zeta ; \lambda)+i \operatorname{Im} f(\hat{x}, \zeta ; \lambda)
$$

so that (5.9) reads eventually

$$
\operatorname{det}\left[\operatorname{Re}^{2} f(\hat{x}, \zeta ; \lambda)-\operatorname{Im}^{2} f(\hat{x}, \zeta ; \lambda)-\sigma_{L}^{2}(\Lambda)+2 i \operatorname{Re} f(\hat{x}, \zeta ; \lambda) \operatorname{Im} f(\hat{x}, \zeta ; \lambda)\right] \neq 0
$$

In particular, when

$$
\operatorname{Im} f(\hat{x}, \zeta ; \lambda)=0
$$

condition (5.11) reduces to

$$
\operatorname{det}\left[\operatorname{Re}^{2} f(\hat{x}, \zeta ; \lambda)-\sigma_{L}^{2}(\Lambda)\right] \neq 0
$$

A sufficient condition for strong ellipticity with respect to the cone $\mathcal{C}-\mathbf{R}_{+}$is therefore the negative-definiteness of $\sigma_{L}^{2}(\Lambda)$ :

$$
\sigma_{L}^{2}(\Lambda)<0
$$

so that

$$
\operatorname{Re}^{2} f(\hat{x}, \zeta ; \lambda)-\sigma_{L}^{2}(\Lambda)>0
$$

and hence (5.13) is fulfilled.

In the derivation of the sufficient conditions (5.11) and (5.14), the assumption (5.5) plays a crucial role. In general, however, $\Pi$ and $f$ have a non-vanishing commutator, and hence a $C(\hat{x}, \zeta ; \lambda)$ exists such that

$$
\Pi(\hat{x}) f(\hat{x}, \zeta ; \lambda)-f(\hat{x}, \zeta ; \lambda) \Pi(\hat{x})=C(\hat{x}, \zeta ; \lambda) .
$$


The occurrence of $C$ is a peculiar feature of the fully pseudo-differential framework. Equation (5.3) is then equivalent to (now we write explicitly also the independent variables in the leading symbol of $\Lambda$ )

$$
\begin{aligned}
& {\left[\left(\sigma_{L}(\Lambda)-C\right)(\hat{x}, \zeta ; \lambda)+f(\hat{x}, \zeta ; \lambda)\right] \varphi(0, \hat{x}, \zeta ; \lambda)} \\
& =\rho^{\prime}(0, \hat{x}, \zeta ; \lambda)-C(\hat{x}, \zeta ; \lambda) \rho(0, \hat{x}, \zeta ; \lambda) .
\end{aligned}
$$

On defining

$$
\gamma(\hat{x}, \zeta ; \lambda) \equiv\left[\sigma_{L}(\Lambda)-C\right](\hat{x}, \zeta ; \lambda)
$$

we therefore obtain strong ellipticity conditions formally analogous to (5.7) or (5.11) or (5.13), with $\sigma_{L}(\Lambda)$ replaced by $\gamma(\hat{x}, \zeta ; \lambda)$ therein, i.e.

$$
\operatorname{det}[\gamma(\hat{x}, \zeta ; \lambda)+f(\hat{x}, \zeta ; \lambda)] \neq 0 \forall \lambda \in \mathcal{C}-\mathbf{R}_{+}
$$

which is satisfied if

$$
\operatorname{det}\left[\operatorname{Re}^{2} f(\hat{x}, \zeta ; \lambda)-\operatorname{Im}^{2} f(\hat{x}, \zeta ; \lambda)-\gamma^{2}(\hat{x}, \zeta ; \lambda)+2 i \operatorname{Re} f(\hat{x}, \zeta ; \lambda) \operatorname{Im} f(\hat{x}, \zeta ; \lambda)\right] \neq 0
$$

\section{Further applications}

In the case of more mathematical interest where the operator in the interior of $M$ remains a Laplacian, while the boundary operator has a pseudo-differential sector, the analysis is much simpler. For example, two cases can be considered.

(i) If $\widetilde{\Lambda}$ is a pseudo-differential operator of order 1 , the leading symbol of the boundary operator (3.9) can be cast in the form (cf. (4.7))

$$
\sigma_{L}(\mathcal{B})=\left(\begin{array}{cc}
\Pi & 0 \\
i(T+\widetilde{T}) & I-\Pi
\end{array}\right)
$$

where $T \equiv \Gamma^{j} \zeta_{j}$ and $\widetilde{T}$ results from the occurrence of $\widetilde{\Lambda}$. The sufficient condition for finding solutions of Eq. (4.5') for all $\psi^{\prime}$ reads now

$$
(T+\widetilde{T})^{2}+|\zeta|^{2} I>0 \quad \forall \zeta \neq 0
$$


because one can simply replace $T$ with $T+\widetilde{T}$ in the analysis of Ref. [9], if Eq. (6.1) holds. Thus, if $\widetilde{\Lambda}$ is chosen in such a way that

$$
(T+\widetilde{T})^{2}(\{\hat{x}\},\{\zeta\})>0 \forall \zeta \neq 0
$$

Eq. (4.5') can always be solved with arbitrary $\psi^{\prime}(\varphi)$. The condition (6.3) can be made explicit after re-writing the DeWitt supermetric (3.6) in the more general form

$$
E^{a b c d} \equiv \frac{1}{2}\left(g^{a c} g^{b d}+g^{a d} g^{b c}\right)+\alpha g^{a b} g^{c d}
$$

Thus, on defining (with $e_{a}^{i}$ being a local tangent frame on $\partial M$ )

$$
\zeta_{a} \equiv e_{a}^{j} \zeta_{j}
$$

and introducing the nilpotent matrices

$$
\begin{aligned}
& \left(p_{1}\right)_{a b}{ }^{c d} \equiv N_{a} N_{b} \zeta^{(c} N^{d)}, \\
& \left.\left(p_{2}\right)_{a b}{ }^{c d} \equiv N_{(a} \zeta_{b}\right) N^{c} N^{d},
\end{aligned}
$$

the work in Ref. [9] finds the useful formula

$$
T=-\frac{1}{(1+\alpha)} p_{1}+p_{2}
$$

and this should be inserted into (6.3) to restrict the kernel of $\widetilde{\Lambda}$, whose leading symbol is equal to $i \widetilde{T}$. The resulting restriction on $\alpha$ should be made compatible with the values of $\alpha$ for which the ellipticity condition (4.14) is fulfilled in the interior of $M$. From this point of view, one has definitely more choice than in the case of the local boundary operator (2.2) for an operator of Laplace type on metric perturbations, because the values of $\alpha$ for which the condition

$$
T^{2}+|\zeta|^{2} I>0 \quad \forall \zeta \neq 0
$$

holds (cf. (6.2)) are incompatible with the occurrence of an operator of Laplace type on metric perturbations [9]. 
(ii) If $\widetilde{\Lambda}$ is a pseudo-differential operator of order $d>1$ (but not necessarily integer), the leading symbol of the boundary operator (3.9) can be expressed in the form

$$
\sigma_{L}(\mathcal{B})=\left(\begin{array}{cc}
\Pi & 0 \\
\widehat{T} & I-\Pi
\end{array}\right) .
$$

The sufficient condition for finding solutions of Eq. (4.5') reads instead

$$
-\widehat{T}^{2}+|\zeta|^{2} I>0 \quad \forall \zeta \neq 0
$$

It is therefore sufficient to choose $\widetilde{\Lambda}$ in such a way that

$$
\widehat{T}^{2}<0 \forall \zeta \neq 0
$$

\section{Concluding remarks}

The mathematical literature and, in particular, the work by Grubb [14], had already considered boundary conditions of the form $(3.8)$, where $(\Lambda+\widetilde{\Lambda})$ is allowed to be a pseudodifferential operator, but for elliptic differential operators. In physics, however, the requirement of gauge invariance of the boundary conditions for quantum gravity leads to an operator on metric perturbations (see (4.8)-(4.11)) which is itself pseudo-differential, since (3.8) is obtained from the vanishing of the gauge-averaging functional at the boundary (see (3.4)). Our physical problem remains therefore original with respect to the mathematical investigations $[14,15]$. Our main contributions are as follows.

(1) The projector condition for the boundary operator in Euclidean quantum gravity at one loop has been derived in the form (3.15).

(2) A careful definition of strong ellipticity of pseudo-differential boundary-value problems in Euclidean quantum gravity has been proposed in section 4, with detailed physical applications in section 5 (see (5.7), (5.11), (5.13), (5.19) and (5.20)), and further mathematical examples in section 6 . 
In other words, we have provided a complete characterization of the properties of the symbol of the boundary operator for which a set of boundary conditions completely invariant under infinitesimal diffeomorphisms are compatible with a strongly elliptic one-loop quantum theory. The analysis of section 5 is detailed but general, and hence has the merit (as far as we can see) of including all pseudo-differential boundary operators for which the sufficient conditions derived therein can be imposed.

It would be now very interesting to prove that, by virtue of the pseudo-differential nature of $\mathcal{B}$ in (3.9), the quantum state of the universe in one-loop semiclassical theory can be made of surface-state type [12]. This would describe a wave function of the universe with exponential decay away from the boundary, which might provide a novel description of quantum physics at the Planck length. It therefore seems that by insisting on pathintegral quantization, strong ellipticity of the Euclidean theory and invariance principles, new deep perspectives are in sight. These are in turn closer to what we may hope to test, i.e. the one-loop semiclassical approximation in quantum gravity [17]. In the seventies, such calculations could provide a guiding principle for selecting couplings of matter fields to gravity in a unified field theory [18]. Now they can lead instead to a deeper understanding of the interplay between non-local formulations [19-21], elliptic theory [22,23], gauge-invariant quantization [13] and a quantum theory of the very early universe [17].

\section{Acknowledgment}

This work has been partially supported by PRIN97 "Sintesi". Correspondence and conversations with Gerd Brubb have been very helpful. The author is indebted to Ivan Avramidi for previous collaboration, and to Pietro Santorelli for encouragement.

\section{References}

1. S. W. Hawking and G. F. R. Ellis, The Large-Scale Structure of Space-Time, Cambridge University Press, Cambridge, 1973.

2. E. Witten, Not. Amer. Math. Soc. 45 (1998) 1124.

3. B. S. DeWitt, Dynamical Theory of Groups and Fields, Gordon and Breach, New York, 1965. 
4. B. S. DeWitt, in Relativity, Groups and Topology II, edited by B. S. DeWitt and R. Stora, North-Holland, Amsterdam, 1984.

5. R. P. Feynman, Rev. Mod. Phys. 20 (1948) 367.

6. C. W. Misner, Rev. Mod. Phys. 29 (1957) 497.

7. S. W. Hawking, in General Relativity, an Einstein Centenary Survey, edited by S. W. Hawking and W. Israel, Cambridge University Press, Cambridge, 1979.

8. P. B. Gilkey, Invariance Theory, the Heat Equation and the Atiyah-Singer Index theorem, Chemical Rubber Company, Boca Raton, 1995.

9. I. G. Avramidi and G. Esposito, Commun. Math. Phys. 200 (1999) 495.

10. G. Esposito, Class. Quantum Grav. 16 (1999) 1113.

11. G. Esposito, Class. Quantum Grav. 16 (1999) 3999.

12. M. Schröder, Rep. Math. Phys. 27 (1989) 259.

13. G. Esposito and C. Stornaiolo, Int. J. Mod. Phys. A15 (2000) 449.

14. G. Grubb, Ann. Scuola Normale Superiore Pisa, Ser. IV, 1 (1974) 1.

15. P. B. Gilkey and L. Smith, J. Diff. Geom. 18 (1983) 393.

16. G. Grubb, Functional Calculus of Pseudodifferential Boundary Problems, Birkhäuser, Boston, 1996.

17. G. Esposito, A. Yu. Kamenshchik and G. Pollifrone, Euclidean Quantum Gravity on Manifolds with Boundary, Fundamental Theories of Physics, Vol. 85, Kluwer, Dordrecht, 1997.

18. G. 't Hooft and M. Veltman, Ann. Inst. H. Poincaré 20 (1974) 69.

19. J. W. Moffat, Phys. Rev. D41 (1990) 1177.

20. D. Evens, J. W. Moffat, G. Kleppe and R. P. Woodard, Phys. Rev. D43 (1991) 499.

21. V. N. Marachevsky and D. V. Vassilevich, Class. Quantum Grav. 13 (1996) 645.

22. I. G. Avramidi and G. Esposito, Class. Quantum Grav. 15 (1998) 281.

23. J. S. Dowker and K. Kirsten, Class. Quantum Grav. 16 (1999) 1917. 